\title{
End Quotes
}

I have a feeling that one can tweak the model to make it fit. D. Hartwick

This is certainly not an exhausting list of references (of carbon star surveys). C. Loup

If you put both diagrams on top of each other, I think that you will agree that it is a scientific, as well as an artistic, mess. M. Dopita

If someone asks me nicely, I will run some models. M. Dopita

He has kind of given me permission to report this work. L. Gardiner

A question from the audience. May I say something? Reply by D. Welch. I'm sure you will say something anyway.

When I say they are not there, I really mean they are not there. G. Da Costa

These numbers include errors and not opinions. G. Bono

No one will be satisfied with plausible reasons: what we want are real reasons. M. Feast

Sorry Nino, no one is interested in the ring distances anymore. D. Welch

All this rubbish ... is just rubbish. N. Panagia

Afterwards, anyone can pitch in, ... provided they are polite. M. Feast.

We thank our speaker from Eastern France. J. Gallagher to H. Zinnecker (Prompted by the rather unusual World Cup outfit of the speaker in honour of the win by France in the World Cup).

I asked the question in their exam and they couldn't answer it. But I couldn't answer it either, so I passed them. J. Dickel

Anyone who has been in astronomy long enough knows that differencing large numbers with systematic errors yields differences with systematic errors. J. Gallagher

I'm under attack here. J. Gallagher

The star formation rate in the LMC is consistent with the HST data. J. Gallagher

Microlensing is just an excuse to do great stellar work. S. Beaulieu

When Cassinelli proposed that there were $2000 M_{\odot}$ stars in R136, theoreticians could suddenly produce stable models. P. Massey

Abundance analyses are where stars earn their keep. K. Venn

The core kinda goes "poof". V. Smith describing an aspect of AGB evolution. 
This isn't the real world and I'm an astronomer. V. Smith

Unfortunately, the thesis was written in German, but at least I can read the tables. D. Garnett

These are the conclusions which you will have to read because the session chair is approaching rapidly ... N. Walborn

At first Phil thought he kept on getting the same spectrum from the HST archives. D. Hunter

Most of my science fiction is read in the Astrophysical Journal. P. Hodge (editor of the Astronomical Journal)

But it is certain that it is not known to any one man to know all things... (a quote on a viewgraph). Certainly he did not know Jay Gallagher. P. Hodge

I have no prejudices - only inclinations. P. Hodge

... and last, and also least, planets. P. Hodge

First you eliminate all the uninteresting objects in the $L M C$ - the stars. C. Smith

The ISM in the LMC is a mess. K. de Boer

Introductory remarks, like skirts, are better shorter. Y.-H. Chu

If I am rushed, it is only because my thesis advisor will get mad at me if I overrun the five minutes. R. Williams

It is easier to talk about what you do than what others do, because you understand what you do. J. Bregman

One little clump of stars does not an $O B$ association make. P. Massey

Starburst is a phrase which, as far as I can tell, is used to describe any star formation more vigorous than Orion. P. Massey 